\title{
An s-domain model of an HVdc converter
}

\author{
S. Todd \\ A. R. Wood \\ MIEEE \\ P. S. Bodger \\ Non-member
}

\begin{abstract}
This paper describes a technique to model an HVdc converter installation in the s-domain. A system equivalent is developed, utilising a frequency domain model, allowing the closed loop frequency response to be expressed. A rational function approximation of the closed loop frequency response is found by writing an overdetermined equation set, solved directly using Singular Value Decomposition. System poles and zeros in the s-domain are extracted through the solution of the rational function roots. Validation of the model at two converter operating points is obtained via time domain simulation and step response techniques for an inverter voltage perturbation.
\end{abstract}

\section{INTRODUCTION}

HVdc converters are unique in power transmission systems, by virtue of their large capacity and fast controllability. During the thirty years of their successful operation, HVdc control systems have been developed to a high level of sophistication. However, it has proven difficult to model the HVdc converter in a way that allows access to the full array of design tools available to a modern control engineer. The lack of suitable models in a control domain makes it much more difficult to achieve the optimum performance from a given installation.

The first accurate models of $\mathrm{AC} / \mathrm{DC}$ interactions through an HVdc converter were based on simulators, now largely superseded by time domain digital simulation. These time domain models are reliable and easy to use, and as such allow verification of plant operation under any number of different operating conditions. As a test-

PE-296-PWRD-0-01-1997 A paper recommended and approved by the IEEE Transmission and Distribution Committee of the IEEE Power Engineering Society for publication in the IEEE Transactions on Power Delivery. Manuscript submitted July 24, 1996; made available for printing January 8, 1997. bed they are unparalleled, however they don't provide the analytical insight needed for optimal system design.

The first analytical approach to the representation of an HVdc converter in a control domain was by Persson in 1970 [1]. In this classic paper the converter distortion interactions were modelled using transfer functions, allowing the generation of Nyquist plots for system stability analysis. This model was quite advanced, including both the effect of the commutation period and DC current distortion in the transfers.

In 1974 Sucena-Paiva and Freris [2] took a describing function approach to model the transfer of waveform distortion through the converter, again using Nyquist analysis for stability studies. While the model was simpler, good accuracy was achieved.

Larsen et al [3] used numerically derived describing functions to model the linear relationships between harmonics at the converter terminals, and proposed a three port network in matrix form to represent the harmonic transfers. Included was the effect of the AC and DC system impedances and converter control system.

Recently Wood and Arrillaga [4] proposed a transfer function model in the frequency domain based on the describing function approach. It approximated the converter as a three-port network and included the effect of commutation period variation, firing angle modulation and converter control. While containing some approximations, good validation of predicted distortion transfer with dynamic simulation was obtained.

This model allowed the successful identification and control of a $70 \mathrm{~Hz}$ composite resonance problem [5] apparent in the CIGRE Benchmark HVdc test system [6]. The system sensitivity to current controller gain was highlighted, and a simple optimisation of the control parameters completed, providing suitable damping at the composite resonant frequency.

This paper describes the development of an $s$-domain model for an HVdc converter and its associated AC and DC systems, based on the transfer function model developed by Wood and Arrillaga. The closed loop system poles and zeros are extracted from the model and used to predict the transient response, which is validated by dynamic simulation for two converter operating points. A simplified CIGRE Benchmark HVdc test system, with the 
inverter modelled as a voltage source, is used throughout.

\section{HVDC SYSTEM TRANSFER FUNCTION DEVELOPMENT}

The frequency domain model used in this work is based on a transfer function approach [5]. Algebraic transfer functions are written to describe the switching patterns of the converter thyristors, and used to define the transfer of voltage from the $\mathrm{AC}$ side to the $\mathrm{DC}$ side, and current from the DC side to the AC side. The transfer functions are themselves dependent on system waveform distortion through the converter controller and commutation period dynamics, and these important effects are incorporated.

Although many frequencies are generated, system dynamics are governed by sets of three coupled frequencies, described by the converter three port model [3]. The converter model utilised in this work is restricted to the three port coupling.

Through reduction to the DC voltage and current variables, the three port model is utilised to represent the rectifier and associated $\mathrm{AC}$ system, as a driving point impedance transfer function $Z_{\text {convr }}$ on the $\mathrm{DC}$ side. This can be regarded as the combined frequency response of these components.

DC current variations contribute to the converter DC side voltage through constant current control action. This can be described as an impedance, and is incorporated in the total converter impedance $Z_{\text {convr }}$. The controller contribution is important for system dynamics.

Coupled with a DC line frequency domain defined impedance, or in this case the simplified CIGRE Benchmark model DC line, an electrical equivalent can be expressed. This is shown in Fig.1.

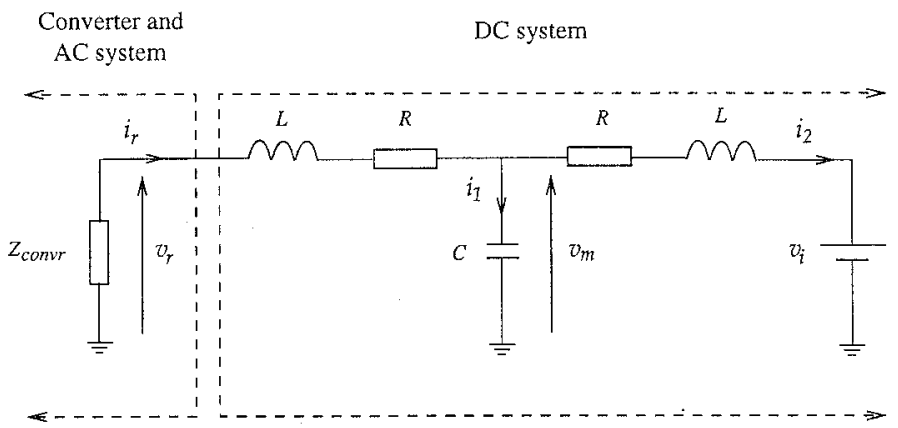

Fig. 1. Electrical equivalent of the simplified CIGRE Benchmark HVdc test system

Although the inverter has been simplified to a voltage source, its impedance can also be easily represented [7] for a complete system solution.

The electrical equivalent voltage and current interactions allow a transfer function block diagram to be derived, which can determine the system closed loop frequency response.
The inverter voltage source $v_{i}$, is defined along with the DC line mid-point voltage $v_{m}$, DC line capacitor current $i_{1}$, and inverter DC current $i_{2}$, allowing equations that govern the steady state voltage and current interactions to be written.

$$
\begin{aligned}
I_{r} & =I_{1}+I_{2} \\
V_{r}-V_{m} & =I_{r}(R+j \omega L) \\
V_{m}-V_{i} & =I_{2}(R+j \omega L) \\
V_{m} & =I_{1} \frac{1}{j \omega C} \\
\frac{V_{r}}{-I_{r}} & =Z_{\text {convr }}
\end{aligned}
$$

Eliminating $V_{m}, I_{1}$ and $I_{2}$ from Eq.1 to Eq.4, results in transfer functions $G_{a}$ and $G_{b}$, that relate the DC voltages, $V_{r}$ and $V_{i}$, to the rectifier DC current, $I_{r}$.

$$
I_{r}=G_{a} V_{r}-G_{b} V_{i}
$$

where

$$
\begin{aligned}
& G_{a}=\frac{\left(1-\omega^{2} L C\right)+j(\omega C R)}{\left(2 R-\omega^{2} 2 L C R\right)+j\left(\omega C R^{2}+\omega 2 L-\omega^{3} L^{2} C\right)} \\
& G_{b}=\frac{1}{\left(2 R-\omega^{2} 2 L C R\right)+j\left(\omega C R^{2}+\omega 2 L-\omega^{3} L^{2} C\right)}
\end{aligned}
$$

Both Eq. 5 and Eq. 6 are incorporated to form a block diagram transfer function equivalent (Fig.2) with $V_{i}$ modelled as the perturbing input and $I_{r}$, the output.

Other combinations are possible by re-arranging the system representation.

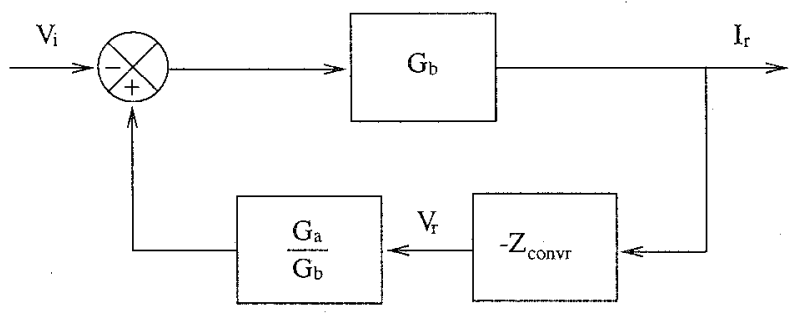

Fig. 2. Transfer function block diagram equivalent of the HVdc system

From Fig.2 the closed loop transfer function can be expressed using block diagram algebra for feedback systems.

$$
\frac{I_{r}}{V_{i}}=\frac{G_{b}}{\left(1+G_{a} Z_{\text {convr }}\right)}
$$

While it is possible to express $G_{a}$ and $G_{b}$ in both the $s$ and frequency domains, $Z_{\text {convr }}$ is defined only in the frequency domain. The resultant closed loop transfer function $\frac{I_{r}}{V_{i}}$, is therefore only defined in the frequency domain. 
The frequency domain model equations [5] coupled with the closed loop transfer function $\frac{I_{r}}{V_{i}}$, are used to determine the system frequency response at a particular converter operating point. This frequency response is dependent on many factors, including the DC current, $\mathrm{AC}$ system voltage, steady state firing angle, commutation angle, and current controller parameters. The AC system impedance in this instance is regarded as constant.

The parameters for two test cases are outlined in the Appendix, with the frequency responses shown in Fig.3 and Fig.4, illustrating the variation of system dynamics with operating point.
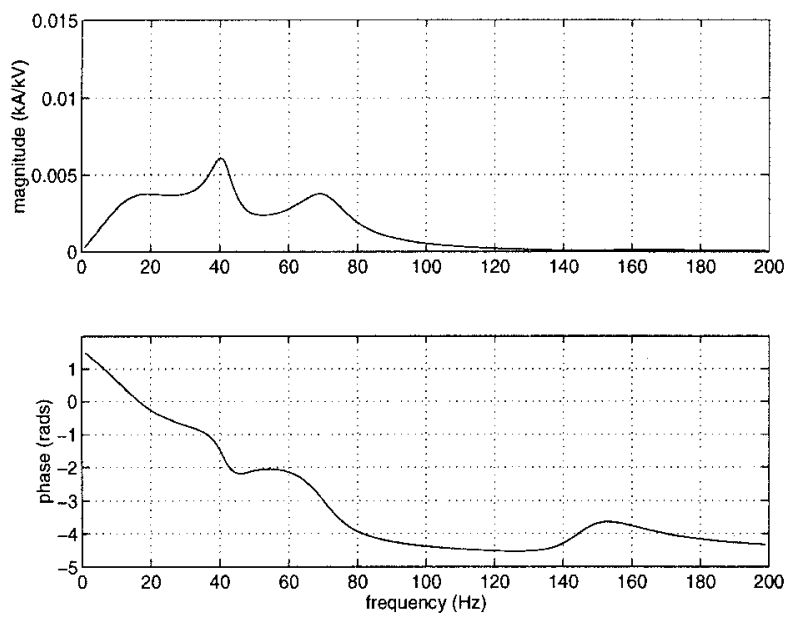

Fig. 3. Closed loop frequency response of the simplified CIGRE system at the $1 k A$ operating point
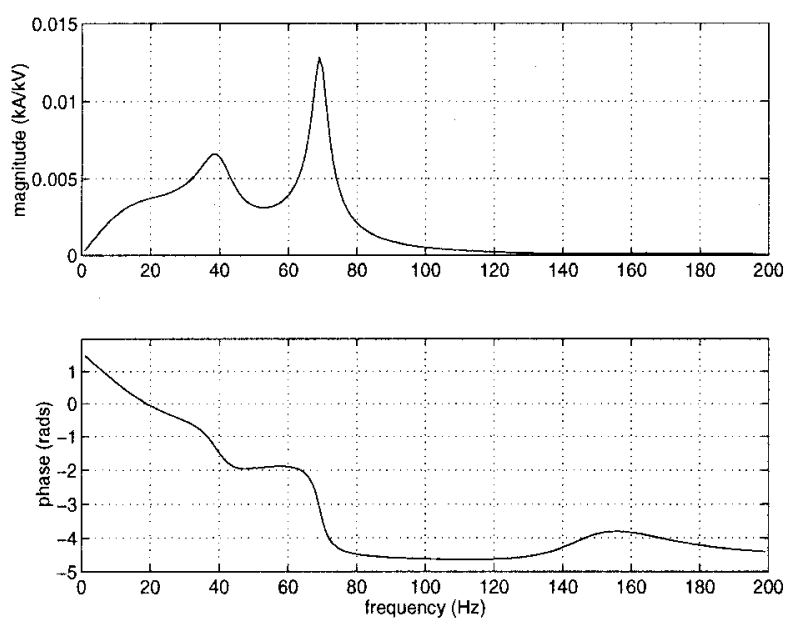

Fig. 4. Closed loop frequency response of the simplified CIGRE system at the $2 k A$ operating point

Although the closed loop transfer function is defined in the frequency domain, system dynamics in the $s$-domain, which will reveal the system transient response, can be extracted by rational function approximation, with the roots directly specifying the closed loop poles and zeros.

\section{RATIONAL FUNCTION APPROXIMATION OF A FREQUENCY RESPONSE}

\section{A. Utilising integral transforms}

Linear or linearised system dynamics can be represented by differential equations in the time domain. The Laplace Transform can greatly simplify their solution by reducing them to algebraic equations in the $s$-domain using the complex operator $s(s=\sigma+j \omega)$. This generally results in rational transfer functions between defined system input and output variables.

The Fourier Transform is a special case of the Laplace Transform, employed to define the steady state response of a continuous system when $s=j \omega$, in the frequency domain.

The reversible nature of the Laplace and Fourier integral transforms between the time, $s$ and frequency domains [8], means a frequency response can be approximated by a rational function in the $s$-domain, with roots revealing system poles and zeros.

Substituting $j \omega$ for $s$, the rational function approximation $\hat{G}(s)$, of numerator and denominator orders $m$ and $n$ respectively, is defined in the frequency domain.

$$
\hat{G}(j \omega)=\frac{b_{0}+b_{1}(j \omega)+b_{2}(j \omega)^{2}+\ldots+b_{m}(j \omega)^{m}}{1+a_{1}(j \omega)+a_{2}(j \omega)^{2}+\ldots+a_{n}(j \omega)^{n}}
$$

\section{B. Linear approximation}

An approach to the rational function approximation problem is to minimise an error function $E$, the difference between $\hat{G}(j \omega)$ and the frequency response $G(j \omega)$ in the least squares sense over the frequency range $k$, in terms of the rational function coefficients, $b_{0}, b_{1}, \ldots, b_{m}$ and $a_{1}, a_{2}, \ldots, a_{n}$.

$$
E=\sum_{k=0}^{p}\left|G\left(j \omega_{k}\right)-\frac{b_{0}+b_{1}\left(j \omega_{k}\right)+\ldots+b_{m}\left(j \omega_{k}\right)^{m}}{1+a_{1}\left(j \omega_{k}\right)+\ldots+a_{n}\left(j \omega_{k}\right)^{n}}\right|^{2}
$$

The derivative information is readily available both analytically and numerically, allowing gradient minimisation routines to be utilised. However coefficients $a_{1}, a_{2}, \ldots, a_{n}$ occur in a non-linear fashion within $E$, requiring a computationally intensive optimisation method [9]. For higher rational function orders this will become prohibitive.

Stahl [10] proposed rewriting the approximation as a set of linear equations, which can be solved directly using linear algebra. The frequency response data $G\left(j \omega_{k}\right)$ is decomposed into real and imaginary components, $c_{k}$ and $d_{k}$, 
and equated with $\hat{G}\left(j \omega_{k}\right)$ for each frequency data point $\omega_{k}, k=0,1, \ldots, p$.

$$
\left(c_{k}+j d_{k}\right)=\frac{b_{0}+b_{1}\left(j \omega_{k}\right)+\ldots+b_{m}\left(j \omega_{k}\right)^{m}}{1+a_{1}\left(j \omega_{k}\right)+\ldots+a_{n}\left(j \omega_{k}\right)^{n}}
$$

This is further written as

$$
\left(c_{k}+j d_{k}\right)=\frac{\left(b_{0}-\omega_{k}^{2} b_{2}+\ldots\right)+j\left(\omega_{k} b_{1}-\omega_{k}^{3} b_{3}+\ldots\right)}{\left(1-\omega_{k}^{2} a_{2}+\ldots\right)+j\left(\omega_{k} a_{1}-\omega_{k}^{3} a_{3}+\ldots\right) .}
$$

To allow all rational function coefficients to occur linearly within the equation set, the problem is expanded

$$
\begin{gathered}
\left(c_{k}+j d_{k}\right)\left[\left(1-\omega_{k}^{2} a_{2}+\ldots\right)+j\left(\omega_{k} a_{1}-\omega_{k}^{3} a_{3}+\ldots\right)\right]= \\
{\left[\left(b_{0}-\omega_{k}^{2} b_{2}+\ldots\right)+j\left(\omega_{k} b_{1}-\omega_{k}^{3} b_{3}+\ldots\right)\right] .}
\end{gathered}
$$

Equating the real and imaginary parts, two sets of linear equations can be written. For the real part

$$
\begin{array}{r}
-d_{k} \omega_{k} a_{1}-c_{k} \omega_{k}^{2} a_{2}+d_{k} \omega_{k}^{3} a_{3}+c_{k} \omega_{k}^{4} a_{4}-\ldots \\
\ldots-b_{0}+\omega_{k}^{2} b_{2}-\omega_{k}^{4} b_{4}+\ldots=-c_{k}
\end{array}
$$

and imaginary part

$$
\begin{array}{r}
c_{k} \omega_{k} a_{1}-d_{k} \omega_{k}^{2} a_{2}-c_{k} \omega_{k}^{3} a_{3}+d_{k} \omega_{k}^{4} a_{4}+\ldots \\
\ldots-\omega_{k} b_{1}+\omega_{k}^{3} b_{3}-\omega_{k}^{5} b_{5}+\ldots=-d_{k} .
\end{array}
$$

The equations can be expressed in matrix form $A x=b$, for $k$ frequency points, with the coefficients of the rational function defined in $x$.

$$
\begin{gathered}
\mathrm{A}=\left(\begin{array}{cc}
B_{i j} & D_{i j^{\prime}} \\
C_{i j} & E_{i j^{\prime}}
\end{array}\right) \\
x=\left(a_{1}, \ldots, a_{n}, b_{0}, \ldots, b_{m}\right)^{T}, \\
\mathrm{~b}=\left(-c_{0}, \ldots,-c_{k},-d_{0}, \ldots,-d_{k}\right)^{T},
\end{gathered}
$$

where rows $i=k+1$, and columns $j=n$ and $j^{\prime}=m+1$. The components in A can be written in terms of $c_{k}, d_{k}$ and $\omega_{k}$ as,

$$
\begin{gathered}
B_{i j}=\sin \left(\frac{-j \pi}{2}\right) w_{i-1}^{j} d_{i-1}+\cos \left(\frac{j \pi}{2}\right) w_{i-1}^{j} c_{i-1} \\
C_{i j}=\cos \left(\frac{j \pi}{2}\right) w_{i-1}^{j} d_{i-1}+\sin \left(\frac{j \pi}{2}\right) w_{i-1}^{j} c_{i-1}
\end{gathered}
$$

$$
\begin{aligned}
& D_{i j^{\prime}}=\cos \left(\frac{-j^{\prime} \pi}{2}\right) w_{i-1}^{j^{\prime}-1}, \\
& E_{i j^{\prime}}=\sin \left(\frac{-j^{\prime} \pi}{2}\right) w_{i-1}^{j^{\prime}-1} .
\end{aligned}
$$

Generally an approximation over many data points will result in $\mathrm{A} x=\mathrm{b}$ being overdetermined, requiring a nonstandard matrix inversion technique. Singular Value Decomposition (SVD) was chosen due to its stability and least squares (LS) solution capability [11].

\section{Matrix condition}

Matrix condition in overdetermined equation sets is a measure of the magnitude ratio of the largest valued singular value to the smallest, and indicates the numerical confidence in a solution.

When approximating data using polynomial power series as basis functions, matrix condition problems may arise, particularly when the dynamic range is large and approximation orders high. This problem was encountered, however Euclidean scaling was successfully employed as a reconditioning technique.

Generally, given a matrix $\mathrm{A}_{p q}$

$$
\mathrm{A}_{p q}=\left(\begin{array}{cccc}
a_{11} & a_{12} & \ldots & a_{1 q} \\
a_{21} & a_{22} & \ldots & a_{2 q} \\
\vdots & \vdots & \ddots & \vdots \\
a_{p 1} & a_{p 2} & \ldots & a_{p q}
\end{array}\right)
$$

The Euclidean length $E_{1}$ for the first column of $\mathrm{A}$ is

$$
E_{1}=\left\|\mathrm{A}_{p 1}\right\|=\sqrt{a_{11}^{2}+a_{21}^{2}+\ldots+a_{p 1}^{2}}
$$

The first column vector $A_{p 1}$ is rescaled by $E_{1}$ to create a new column vector $\hat{\mathrm{A}}_{p 1}$

$$
\hat{\mathrm{A}}_{p 1}=\frac{\mathrm{A}_{p 1}}{E_{1}}
$$

A new matrix $\hat{\mathrm{A}}_{p q}$ is constructed from $\mathrm{A}_{p q}$, with each column scaled by its Euclidean length. SVD is applied to the respecified problem, returning a parameter matrix $\hat{x}_{q}$. To return the true rational function coefficients, the terms in $\hat{x}_{q}$ are scaled by each corresponding Euclidean length from $\mathrm{A}_{p q}$.

$\left(x_{1}, x_{2}, \ldots, x_{q}\right)=\left(\hat{x}_{1}, \hat{x}_{2}, \ldots, \hat{x}_{q}\right)\left(\begin{array}{cccc}\frac{1}{E_{1}} & 0 & \ldots & 0 \\ 0 & \frac{1}{E_{2}} & \ldots & 0 \\ \vdots & \vdots & \ddots & \vdots \\ 0 & 0 & \ldots & \frac{1}{E_{q}}\end{array}\right)$ 


\section{Approximation range and model order selection}

The choice of model that approximates a frequency response in the $s$-domain will be unknown unless there is a priori knowledge of the system.

The closed loop frequency response at each operating point indicated no significant dynamics were evident above $200 \mathrm{~Hz}$. As a consequence the approximation frequency range was confined between $0 \mathrm{~Hz}$ and $200 \mathrm{~Hz}$. The converter AC system impedance, and consequently the converter impedance $Z_{\text {convr }}$ was also known to have a sharp parallel resonance. For this reason a $1 \mathrm{~Hz}$ approximation resolution was chosen to define the impedance accurately around the resonant point. This is important if system impedances are strongly frequency dependent near the final system natural frequencies.

For the closed loop HVdc system in Fig.2 the DC line component values are known, allowing $G_{a}$ and $G_{b}$ to be defined in the $s$ and frequency domains. However, $Z_{c o n v r}$ which is defined only in the frequency domain, introduces uncertainty into the pole/zero identification process.

One approach to model order selection using rational functions is to increase the numerator and denominator orders until approximation error reaches a minimum, with roots determining the poles and zeros.

The summed absolute real and imaginary error for $0-200 \mathrm{~Hz}$ with respect to increasing numerator and denominator order for the approximation at the $2 k A$ operating point, is shown in Fig.5.

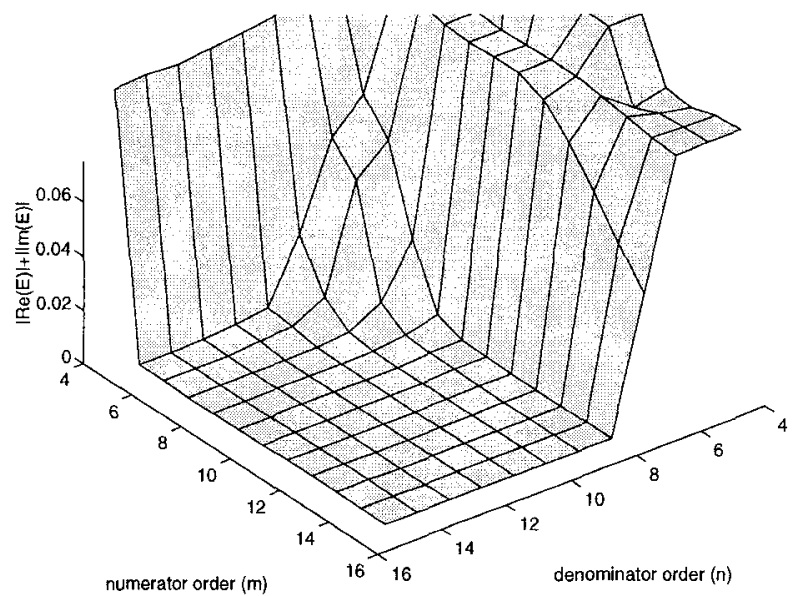

Fig. 5. Summed absolute real and imaginary error $(k A / k V)$ of the rational function approximation for the $2 k A$ operating point, dependent on numerator and denominator order

The error plateaus significantly for $m \geq 8$ and $n \geq 8$, corresponding to high approximation accuracy over the whole frequency range. For the function to be realisable, $n \geq m$ is a requirement.
For orders $m \geq 8$ and $n \geq 8$, the rational function roots consistently returned 5 unique zeros and 8 unique poles in similar positions in the $s$-domain. The remainder could either be discarded due to pole/zero cancellation or were sufficiently outside the approximation bandwidth to be of negligible importance. Any approximation orders below this resulted in large error and little confidence in the roots.

\section{Application and VAlidation}

The roots of the approximating rational function relate to closed loop pole and zero positions in the $s$-domain at a specific converter operating point.

The transient response of the $s$-domain model was obtained by applying a unit step at the inverter voltage $\left(V_{i}\right)$ input (Fig.2) using the Matlab Control Systems Toolbox. For validation purposes this is compared with transient results using the electromagnetic transients package PSCAD/EMTDC, for an inverter voltage 40 per cent step reduction from $488 \mathrm{kV}$ to $300 \mathrm{kV}$, using the simplified CIGRE system.

Validation of the model is presented for two DC current operating points, highlighting the variation of system dynamics.

\section{A. $1 k A$ operating point}

The solution of the roots at the $1 k A$ operating point (Table I) show that all closed loop poles reside in the left hand $s$-plane, illustrating the unconditional stability of the system.

The lightly damped complex conjugate pole pair at $p_{3,4}=-21.87 \pm j 257.50$, indicates the response will contain a $40 \mathrm{~Hz}$ oscillation, while the heavily damped low frequency poles at $p_{1,2}=-70.52 \pm j 75.06$ will contribute an initial $16 \mathrm{H} z$ effect.

\begin{tabular}{|c|c|}
\hline poles & zeros \\
\hline$p_{1,2}=-70.52 \pm j 75.06$ & $z_{1}=0$ \\
$p_{3,4}=-21.87 \pm j 257.50$ & $z_{2,3}=-51.72 \pm j 281.67$ \\
$p_{5,6}=-47.15 \pm j 442.03$ & $z_{4,5}=-51.50 \pm j 909.45$ \\
$p_{7,8}=-107.24 \pm j 964.64$ & \\
\hline \multicolumn{2}{|c|}{ TABLE I } \\
\hline
\end{tabular}

Closed loOP POLE AND ZERO POSITIONS FOR THE RATIONAL FUNCTION APPROXIMATION AT THE $1 k A$ OPERATING POINT

The transient results (Fig.6) compare favorably at this operating point, with the approximate $40 \mathrm{~Hz}$ and lower frequency effect apparent in both the model and the PSCAD/EMTDC simulation. Transient rise time, overshoot and damping are also in agreement. 


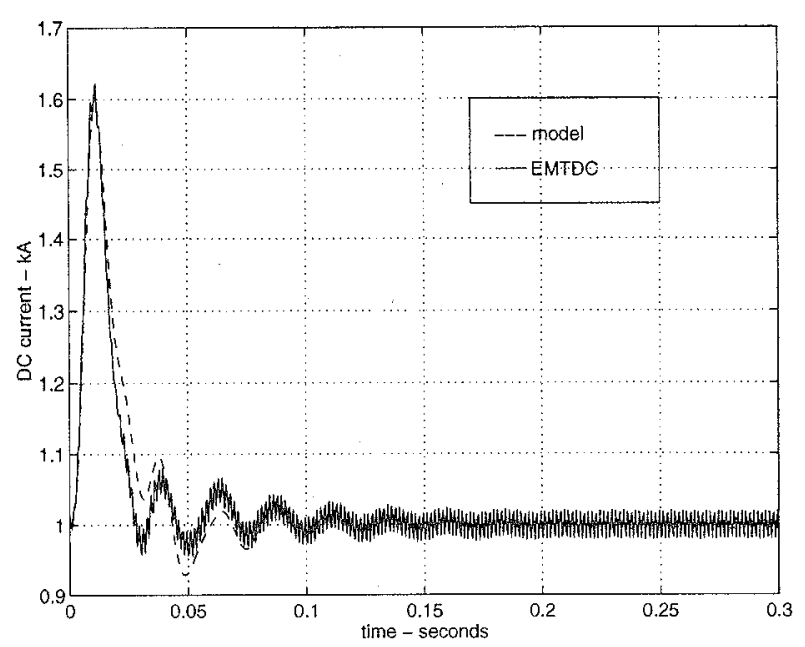

Fig. 6. Comparison of model and PSCAD/EMTDC simulation for $V_{i}$ input perturbation at the $1 k A$ operating point

\section{B. $2 k A$ operating point}

The closed loop poles and zeros determined for this operating point (Table II) indicate two possible modes of oscillation in the transient response, with lightly damped complex conjugate pole pairs existing at approximately $40 \mathrm{~Hz}$ and $70 \mathrm{~Hz}$. Again all the closed loop poles are resident in the left hand s-plane, indicating stability. The more lightly damped pole pair occurring at $70 \mathrm{~Hz}$ is consistent with the composite resonance frequency pointed out by Wood [5], when operating the CIGRE system at $2 k A$ DC current.

\begin{tabular}{|c|c|}
\hline poles & zeros \\
\hline$p_{1,2}=-95.50 \pm j 58.82$ & $z_{1}=0$ \\
$p_{3,4}=-33.71 \pm j 247.56$ & $z_{2,3}=-83.63 \pm j 284.51$ \\
$p_{5,6}=-14.41 \pm j 434.87$ & $z_{4,5}=-81.32 \pm j 910.18$ \\
$p_{7,8}=-138.01 \pm j 997.85$ & \\
\hline
\end{tabular}

CLOSED LOOP POLE AND ZERO POSITIONS FOR THE RATIONAL FUNCTION APPROXIMATION AT THE $2 k A$ OPERATING POINT

In this instance the comparison indicates that rise time, overshoot and settling time are all similar. Both the $40 \mathrm{~Hz}$ and lightly damped $70 \mathrm{~Hz}$ oscillations are evident as well as a low frequency effect.

\section{Summary of results}

The agreement between the $s$-domain model transient predictions and EMTDC based time domain simulations show this approach has some value. It has been revealed that system dynamics do change for different converter

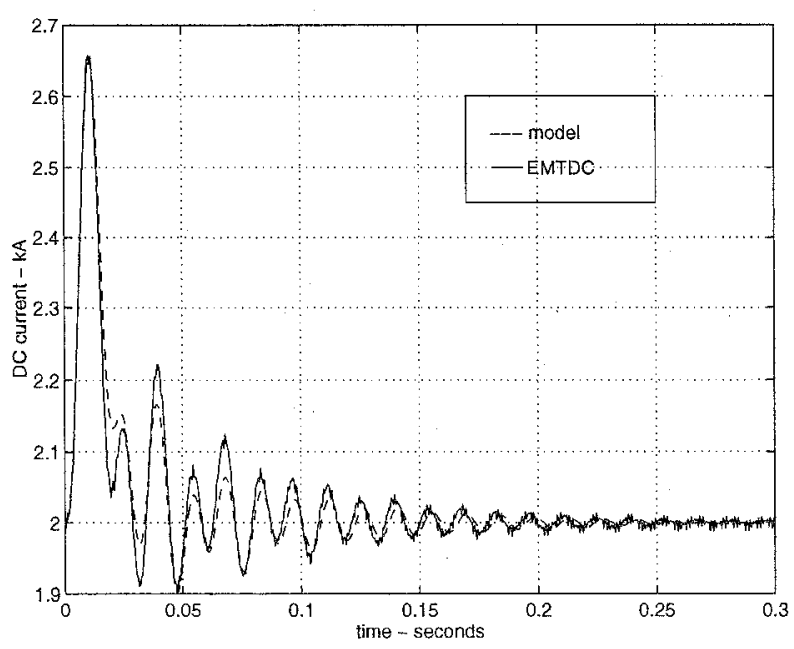

Fig. 7. Comparison of model and PSCAD/EMTDC simulation for $V_{i}$ input perturbation at the $2 k A$ operating point

operating points, indicating the current controller will encounter a range of plant characteristics. The approximation pole/zero results presented in Tables I and II indicate changes in damping for each complex pole/zero pair between operating points, with slight frequency changes also noted in the pole positions.

The small signal approximations of the original frequency domain model are limited to the dynamics of the commutation periods, and were not expected to affect the prediction. This was supported by the agreement in results even for a relatively large perturbation.

The accurate prediction of the transient DC side overcurrents and oscillatory modes indicates the model may be sufficiently robust to be useful for control design purposes. This will be possible when the controller is separately represented within the $s$-domain model, and the dynamic variation over the full operating range, is known.

\section{CONCLUSION}

This paper has presented an approach for modelling a converter installation in the $s$-domain. The frequency domain transfer function model for converter installations developed by Wood et al [4], has been utilised to represent the rectifier, associated AC system and current controller as a driving point impedance on the DC side. This driving point impedance function is regarded as the frequency response for a given $\mathrm{DC}$ current operating point.

A simplified CIGRE Benchmark HVdc test system, with the inverter modelled as a voltage source, is utilised and a transfer function representation of the installation developed in block diagram form. This allows the closed loop frequency response of inverter voltage to rectifier DC current, to be expressed. 
A rational function approximation technique is applied to the closed loop frequency response allowing the determination of the closed loop poles and zeros in the $s$ domain. The approximation is written as an overdetermined linear equation set, and solved directly in the least squares sense using Singular Value Decomposition.

Model selection in the $s$-domain is determined using an error criteria on the approximation in conjunction with the solution of the rational function roots. Extraneous roots are eliminated either through pole/zero cancellation or their existence outside the approximation bandwidth.

The $s$-domain model and PSCAD/EMTDC time domain simulation transient results are validated for an inverter voltage input perturbation for two $\mathrm{DC}$ current operating points. The transient validation of the $s$-domain modelling technique, indicates the future HVdc control design possibilities.

The separation of the current control contribution from within the converter impedance, and work on the optimisation of the controller dynamics is currently in progress.

\section{ACKNowledgements}

The authors wish to thank TRANSPOWER NZ LTD. for the financial support provided to carry out this project.

\section{REFERENCES}

[1] Persson, E.V., "Calculation of transfer functions in grid-controlled convertor systems," IEE Proc., Vol.117, No.5, May 1970, pp989-997.

[2] Sucena-Paiva, J.P. and Freris, L.L., "Stability of a dc transmission link between weak ac systems," IEE Proc., Vol.121, No.6, June 1974, pp508-515.

[3] Larsen, E.V., Baker, D.H. and McIver, J.C., "Loworder harmonic interactions on AC/DC systems," IEEE Trans on Power Delivery, Vol.4, No.1, January 1989, pp493-501.

[4] Wood, A.R. and Arrillaga, J., "HVdc convertor waveform distortion: a frequency domain analysis," IEE Proc. Pt.C, Vol.142, No.1, January 1995, pp88-96.

[5] Wood, A.R. and Arrillaga, J., "Composite resonance; a circuit approach to the waveform distortion dynamics of an $\mathrm{HVdc}$ converter," IEEE Trans on Power Delivery, Vol.10, No.4, October 1995, pp1882-1888.

[6] Szechtmann, M., Weiss, T. and Thio, C.V., "First benchmark model for HVdc control studies," Electra, No.135, April 1991, pp55-75.
[7] Wood, A.R. and Arrillaga, J., "The frequency dependent impedance of an HVdc converter," IEEE Trans on Power Delivery, Vol.10, No.3, July 1995, pp1635-1641.

[8] Brook, D. and Wynne, R.J., "Signal Processing: Principles and Applications," Edward Arnold, 1988, pp78-79.

[9] Whitfield, A.H., "Transfer function synthesis using frequency response data,"

International Journal of Control, Vol.43, No.5, 1986, pp1413-1426.

[10] Stahl, H. "Transfer function synthesis using frequency response data," International Journal of Control, Vol.39, No.3, 1984, pp541-550.

[11] Press, W.H., Teukolsky, S.A., Vetterling, W.T. and Flannery, B.P., "Numerical Recipes in Fortran: The art of scientific computing $2 \mathrm{e}$,"

Cambridge University Press, 1992, pp51-58.

\section{APPENDIX}

\section{Operational parameters}

$\begin{array}{lll}G_{p} & \text { (PI proportional gain) } & -1.0989 \\ T_{i} & \text { (PI integral time const.) } & -0.009 \mathrm{secs} \\ v_{i} & \text { (inverter DC volts) } & -488 k \mathrm{~V} \\ v_{a c_{1}} & (\text { AC volts }(p k-g n d)(1 k A)) & -284.544 k \mathrm{~V} \\ \alpha_{1} & \text { (firing angle }(1 k A)) & -0.452 \mathrm{rads} \\ v_{a c_{2}} & (\text { AC volts }(p k-g n d)(2 \mathrm{kA})) & -282.672 \mathrm{kV} \\ \alpha_{2} & (\text { firing angle }(2 k A)) & -0.303 \mathrm{rads}\end{array}$

\section{Authors}

Simon Todd completed his BE(Hons) at the University of Canterbury, Christchurch, New Zealand, in 1993, where he is presently enrolled as a Ph.D candidate.

Alan Wood completed his BE and Ph.D. at the University of Canterbury, Christchurch, New Zealand, in 1981 and 1993. From 1982 to 1989 he worked for the Electricity corporation of New Zealand, and then for Mitsubishi Electric in the UK and the Middle East. He is presently a lecturer at the University of Canterbury.

Patrick Bodger completed his BE(Hons) and Ph.D at the Unversity of Canterbury in 1972 and 1977 respectively. From 1977 to 1981 he worked for New Zealand Electricity. Since 1982 he has been with the University of Canterbury where he is a senior lecturer. He is a Registered Professional Engineer in New Zealand. 